\title{
Milliliter per Milligram per Meter Squared per Day
}

National Cancer Institute

\section{Source}

National Cancer Institute. Milliliter per Milligram per Meter Squared per Day. NCI

Thesaurus. Code C120821.

A unit of concentration equal to milliliter divided by milligram per meter squared per day. 